G. Münch. But the effect of $\theta^{2}$ Ori A upon the ionization equilibrium of nebular matter in its neighborhood, and hence upon the recombination spectrum there, is not independent of its contribution to the dust-scattered continuum. The reddening produced by the dust on the recombination spectrum can in principle be determined from the recombination lines alone. Therefore, if we know the albedo of the scattering particles, we should be able to relate the scattered spectrum to the recombination spectrum. The integration along the line of sight is always a difficulty, but I fail to see how the observation of scattered light from $\theta^{2}$ Ori A rules out the possibility that this star also contributes to the ionization.

$Y$. Parijsky. Recent radio observations at Pulkovo show that the very center of the Nebula is much brighter than found previously. This means that the density fluctuations in the central region are much smaller. There is no hole in the emission at a scale $\geqslant 0{ }^{\prime}$, nor is there indication of the secondary maximum observed at Stanford.

G. Courtès. Mr P. Cruvellier has recently measured the electron densities over the Orion Nebula from the [O II] doublet ratio, using a highly accurate photo-electric method and FabryPérot dispersion. With the 77 -inch reflector (at the coudé focus), he obtained a spectral resolution of $0.3 \AA$ over a field of $30^{\prime \prime}$. 'The profiles of both $\mathrm{H} \beta$ and the [O II] lines were determined in order to separate temperature and turbulent effects. The large number of measured points permitted the construction of contours of equal electron density, and it was found that these contours follow a smooth elliptical shape, probably bearing a relation to the direction of the magnetic field.

\title{
6. ON THE INTERNAL STRUCTURE OF THE ORION NEBULA
}

\section{K. Wurm}

The investigations which I intend to describe here have the aim of arriving at a better understanding of the optical surface structure of the Orion Nebula. It was hoped to obtain by such a study some idea about the arrangement of the visible masses of the Nebula in space, and also about the positions of the brightest stars in the field relative to these masses.

The Nebula has a very intense kernel. This kernel appears in all monochromatic pictures with equal shape, as well in the stronger emission lines and also in continuous light. There can scarcely be a doubt that the bright kernel owes its shape to absorbing masses in the foreground. However, these absorbing masses belong to the Nebula itself, and they become easily visible in emission by increasing the exposure time. If one compares the distribution of the surface brightness with the distribution of the star density, it becomes clear that there exists a correlation between these two quantities. The star density is highest at places of highest emission density, and low where the emission is weak. That part of the Nebula which I have designated as the bright kernel coincides with the region of high star density. It follows from this property that we are looking deepest into the cluster connected with the Trapezium in the vicinity of this group of stars. The correlation between surface intensity and star density is one of chance. It originates from the distribution of matter at that border of the Nebula which we face from our position on the Earth.

There exists yet a second correlation between two observable quantities (I), namely between the surface brightness in the Balmer lines and the projected electron density measured after the method proposed by Seaton. This correlation is linear. In a recent paper (2), I have shown that this result finds an explanation, if one takes account of the continuous extinction 
in the Nebula due to the dust particles. The surface brightness has in the past always been expressed in the form of the so-called emission measure

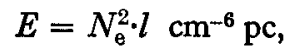

wherein $N_{\mathrm{e}}$ is the electron density and $l$ the linear depth of the Nebula measured in parsecs. It is not difficult to show that the surface intensity has correctly to be represented-assuming a homogeneous dust distribution--by

$$
F=K \frac{N_{\mathrm{e}}}{\sigma}\left(\mathrm{I}-\exp \left[-N_{\mathrm{e}} \sigma x\right]\right)
$$

wherein $K$ is approximately a constant, $\sigma$ the coefficient of extinction counted per hydrogen atom present and $x$ is the linear depth of the Nebula. One sees that for a large optical depth we have:

$$
F=K \frac{N_{\mathrm{e}}}{\sigma}
$$

so $F$ becomes proportional to $N_{\mathrm{e}}$. Since the observations follow clearly the correlation (3), we must conclude: first, that the Nebula is optically thick in all parts which we have measured, and second, that the coefficient $\sigma$ cannot change appreciably from one place to another.

According to equation (3), with a knowledge of the density $N_{\mathrm{e}}$ and the surface intensity $F$ we may derive a numerical figure for the extinction coefficient. The surface intensity $F$ has been found for a spot near the Trapezium to amount to $F_{4,2}=0.124 \mathrm{erg} \mathrm{sec}^{-1} \mathrm{~cm}^{-2} \mathrm{sterad}^{-1}$. This figure includes a correction factor for internebular extinction of $I^{m} \cdot 5$. This value corresponds to an emission measure $E=10^{6.8}$. With an electron density of $N_{\ominus}=10^{4}$ we find $\sigma=5 \times 10^{-22} \mathrm{~cm}^{2}$. If we fix the bottom of the visible layers at $x=2$, we have with $N_{\mathrm{e}} \sigma x=2$, the value $x=0.13 \mathrm{pc}$. This extension for the visible layers is valid for the points of highest surface brightness, and a homogeneous density distribution. At the points of weakest surface brightness, $x$ becomes 100 to 200 times larger.

Since the Trapezium stars show a strong reddening, it was generally concluded that they are deeply involved in the Nebula, and although not distinctly stated it was probably meant that they were imbedded within the visible masses. However, if we have to deal with a Nebula of such a high surface brightness as we find here, much material may be hidden in front of the visible masses.

Concerning in particular the Trapezium stars, I have come to the conclusion that the $\mathrm{He}$ lines of internebular origin appearing in their spectra are formed in an invisible cloud complex in front of the visible masses (3). This result contradicts the presently favoured model (4) in which the Trapezium should be at the bottom of the visible masses. From this model a radial expansion of the central masses relative to the Trapezium has been derived. I bring forward two main arguments against the currently used model and in favour of a position of the stars of the Trapezium in front of the visible masses (3). One, there exists no correlation between the strength of the $\mathrm{He}$ I absorption lines and the surface brightness of the emissions in the vicinity of the stars in which we observe the interstellar He I absorption. Altogether in nine different stars, HeI lines of internebular origin have been observed. The surface brightness near these stars in the [OIII] lines varies by more than a factor 100. This line is the best indicator for favoured conditions for a high population of the metastable level from which the He I absorption line arises. One can conclude from this observation that the appearance of the He I components in the spectra of the Trapezium stars is not bound to the strong central masses in the centre of the Nebula. Moreover, since a high density favours the condition for a high population of the metastable level, one should expect a much higher intensity of the HeI absorption as observed if the Trapezium were situated behind the central bright masses. This can be quantitatively 
shown by a formula which describes the conditions for the dependence of the population on density and other parameters. The second argument against a position of the Trapezium deep in the central bright mass concerns a complete lack of a correlation between the radial velocities derived from the $\mathrm{HeI}$ lines and the radial velocities derived from the emission lines close to the stars in the field. The emission lines have a very pronounced maximum at $+20 \mathrm{~km}$, whereas the HeI components are distributed between $+23 \mathrm{~km}$ and $-3 \mathrm{I} \mathrm{km}$. These two arguments mentioned have together a weight strong enough to force abandonment of the idea that the Trapezium has a position deep in the bright central masses. Hence, a radial expansion of the central masses relative to the Trapezium stars is not supported by observational facts. The masses of the centre are on the contrary moving directly towards the Trapezium.

\section{REFERENCES}

I. Wurm, K., Schuster, H. E. In press, I964.

2. Wurm, K. Z. Astrophys., 58, 99, 1963.

3. Wurm, K. Z. Astrophys., 52, т09, 196r.

4. Wilson, O. C. Münch, G., Flather, E., Coffeen, M. F. $\quad$ Astrophys. F. Suppl., 4, r99, r959.

\section{DISCUSSION}

G. Münch. I agree with Prof. Wurm regarding the incompleteness of the argument built up by Wilson, Münch, Flather, and Coffeen from the radial velocities of the HeI nebular absorption lines. In fact, the reply by Wilson and myself ( $Z$. Astrophys. 56, 127, 1962) to Prof. Wurm's criticism probably is also not tenable, not on evidence provided by the Orion Nebula alone, but also on the basis of observations in other galactic and planetary nebulae. The point is that the observed weakness of the HeI line in all cases, in comparison with theory assuming deactivation by electron collisions and two-photon decay, cannot be explained by the existence of density fluctuations. There must be some other very effective deactivating agent for the $2^{3} \mathrm{~S}$ level of HeI. A brief calculation shows that if the optical depth in Lyman- $\alpha$ is greater than about $10^{4}$, the energy density of $L \alpha$ radiation will be high enough to ionize Her from the $2^{3} \mathrm{~S}$ level much faster than it can be deactivated collisionally. The only evidence favouring this idea at present is that the only planetary nebula where $\mathrm{HeI}_{\mathrm{I}}$ absorption is observed with certainty on the central star is Campbell's hydrogen-envelope star, which has an expansion velocity larger than $100 \mathrm{~km} / \mathrm{sec}$; clearly, such a large expansion velocity favours the escape of trapped $L \alpha$ resonance radiation.

$K$. Wurm. By He I ionization from the metastable $2^{3} \mathrm{~S}$ level, it may be possible to reduce the population by a factor 2 to 5 or even 10 . However, a reduction factor 100 to 1000 , as is required to explain the low $\lambda_{3} 889$ absorption in the Trapezium stars, must be regarded as impossible.

\section{STRUCTURE AND KINEMATICS OF THE ORION NEBULA}

\section{G. Münch}

The state of motion of the gases in the Orion Nebula has been studied from the profiles of the emission lines at a given point in the Nebula, and from the variation in the radial velocities of the lines from point to point over the Nebula. Additional information regarding velocity gradients and stratification is obtained from the comparison of spectrophotometric and radial velocity data for lines of ions with different states of ionization. The observations of line widths carried out at Hamburg-Bergedorf and the radial velocities determined at the Lick Observatory, 